\title{
ASSESSMENT OF FATIGUE AND ITS CORRELATION WITH QUALITY OF LIFE AND FUNCTION DISABILITY IN OSTEOARTHRITIC PATIENTS
}

Naira K.R. Mohamed ${ }^{1}$; Magda R. Zahran ${ }^{2}$ and Abeer A. Yamny ${ }^{3 *}$

1 BSc physical therapy, Faculty of Physical Therapy, Misr University For Science and Technology, Egypt

2- Professor of Physical Therapy, Basic Sciences Department, Faculty of Physical Therapy, Cairo University, Egypt.

3- Professor of Physical Therapy, Basic Sciences Department, Faculty of Physical Therapy, Cairo University, Egypt.

3*Email-abeer.yamany@pt.cu.edu.eg

Key Words: Fatigue, Quality of life, Disability, Knee osteoarthritis, WOMAC, FSS.

\section{ABSTRACT}

Background: Osteoarthritis (OA) is a chronic, degenerative, inflammatory musculoskeletal dysfunction. It is associated with pain, reduced functional capacity and deficient quality of life. Fatigue is a frequent complaint in patients with arthritis, which correlate to tiredness. Purpose of the study: This study aimed to assess fatigue in knee osteoarthritic patients and to correlate it to quality-of-life indexes and functional disability. Subjects and Methods: This cross-sectional study was performed on 200patients of both sex with ages ranged from 50 to 65 years old and medical diagnoses of knee osteoarthritis either grade II or grade III. The participants were selected according to the demographic survey including information on their age, gender, educational level, height, weight, BMI, disease duration. Fatigue was measured by Arabic version of fatigue severity scale; quality of life was measured by Arabic version of SF36 questionnaire and function disability was measured by Arabic version of The Western Ontario and McMaster Universities osteoarthritis index (WOMAC). Results: 200 patients were included in the study. Grade II group consisted of 39 patients ( 3 male and 36female), grade III consisted of 161 patients (31 male and 131female). The results indicated that there was significant difference in the fatigue severity scale between both groups with (Uvalue $=2019, \mathrm{Z}$-value $=\mathbf{- 3 . 4 5 8}, \mathrm{p}=0.001 *$ ), this significant increase in grade III group in compared to grade II group. There was a positive weak correlation between fatigue severity scale and WOMAC $(\mathrm{p}=0.0001 *)$. And There was a negative weak correlation between fatigue and pain domain of SF36 questionnaire $\left(\mathrm{p}=0.0001^{*}\right)$ and there was a negative weak correlation between fatigue severity scale and fatigue domain of SF36 questionnaire $\left(\mathrm{p}=0.002^{*}\right)$. Conclusion: On the basis of this study, it could be concluded that there is an association between fatigue and both disability and quality of life in patients with knee osteoarthritis. 


\section{INTRODUCTION}

Osteoarthritis is the most prevalent form of arthritis. It is a multifactorial process in which mechanical factors have a central role and is characterized by changes in the structure and function of the whole joint(Hunter and Felson, 2006). Rheumatic disease affects older adult populations worldwide and knees are the most commonly affected joints (Dawson et al., 2004). These degenerative changes cause pain, stiffness, and swelling that result in chronic disease and disability with advanced age and seriously alter the quality of life (QoL) (Alkan et al., 2013).

Some risk factors contribute to the appearance of osteoarthritis diseases, such as sex, age, trauma, overuse, and genetic conditions. With disease progression, patients' complaints of physical limitations, pain, and functionality restriction increase leading to an important decrease in their QOL (Kawano et al., 2015)

Fatigue is a common, non-specific, symptom experienced by most people at some point during their lives. It is the enduring, subjective sensation of generalized tiredness or exhaustion. It is also conceptualized variously as weariness, weakness, and depleted energy( Power et al., 2008).

Cultural aspects may play a role in the expression of fatigue (Mortada et al., 2015). Fatigue in OA is not routinely evaluated and has only been considered in a limited number of studies ( Power et al., 2008).

Few studies on fatigue in knee osteoarthritis reported marked levels of fatigue in nearly half of patients (Snijders et al., 2011)( Stebbings et al., 2010). On the other hand, assessment of fatigue in the provision of OA care is often neglected (Power et al., 2008).

Health-related quality of life (HRQoL) is a broad concept representing individual responses to the physical, mental, and social effects of illness or well-being on daily living, which influence the extent to which personal satisfaction with life circumstances can be achieved (Perruccio et al., 2016).

Kawano et al., 2015 showed that patients with knee osteoarthritis have a low perception of their quality of life, especially in the fields of functional capacity, functional limitations, and pain.

Araujo et al., 2016 found a correlation between functional independence and the QOL; the more the functional independence was impaired, the lower the QOL and the severity of osteoarthritis was not correlated with significant loss of QOL. The aim of this study was to investigate the association between fatigue and both functional disability and quality of life in patients with knee osteoarthritis.

\section{SUBJECTS, MATERIALS AND METHODS}

This study was conducted at 6 October Hospital, Health Units and the out clinic of Faculty of Physical Therapy, Cairo University from January 2019 to 
November 2019. The study was approved by ethical comity of Faculty of Physical Therapy, Cairo University with code number: P.T.REC/012/001930

i. Design of the study:

The study design was a cross sectional study

ii. Subjects:

200 patients according to power analysis participated in this study with age ranged from 50 to 65 years old of both males and females with a medical diagnosis of knee osteoarthritis of either grade II and III. All patients singed data in the informed consent form. Patients were recruited from the outpatient clinic on 6 October Hospital, Health Units, and the out clinic of Faculty of Physical Therapy, Cairo University.

Inclusion criteria: patients were included, if they had

- a medical diagnosis of knee osteoarthritis of both side

- Both male and female.

- Aged between 50 and 65 years old.

- BMI: 18.5 to $24.9 \mathrm{~kg} / \mathrm{m}^{2}$.

- grade II and III of knee osteoarthritis according to Kellgren and Lawrence system for classification of osteoarthritis of knee (Kellgren and Lawrence, 1957).

\section{Exclusion criteria:}

Patients were excluded if they had:

- Previous orthopedic or neurological disorders of Lower limb, pelvis or spine.

- Previous surgeries of Lower limb, pelvis or spine.

- Other degenerative disease that makes the patient unable to participate in the study.

- Traumatic conditions of Lower limb, pelvis or spine.

- Deformities of Lower limb, pelvis or spine.

Instruments

1. Demographic survey including information on their age, gender, educational level, height, weight, and disease duration.

2. Fatigue Severity Scale(Arabic version) to assess fatigue which is valid and reliable (Al-Sobayel et al., 2016) .The scale includes 9-items with questions related to how fatigue interferes with certain activities and rates its severity according to a self-report scale. The items are scored on a 7-point scale with $1=$ strongly disagree and $7=$ strongly agree. The minimum score $=9$ and maximum score possible $=63$. Higher the score $=$ greater fatigue severity . Another way of scoring is taking the mean of all the scores with minimum score being 1 and maximum score being 7 .

3. The SF36 questionnaire(Arabic version) to assess quality of life which is valid and reliable (Abdulaziz et al., 1998).

SF-36 is a widely applied instrument for measuring health status and consists of eight scales yielding two summary measures: physical and mental health. The physical health measure includes four scales of physical functioning 
(10 items), role-physical (4 items), bodily pain (2 items), and general health (5 items). The mental health measure is composed of vitality (4 items), social functioning ( 2 items), role-emotional ( 3 items), and mental health (5 items). To score the SF-36, scales are standardized with a scoring algorithm or by the SF$36 \mathrm{v} 2$ scoring software to obtain a score ranging from 0 to 100 . Higher scores indicate better health status, and a mean score of 50 has been articulated as a normative value for all scales(Alkan et al., 2014).

4. The Western Ontario and McMaster Universities osteoarthritis index (WOMAC)(Arabic version) to assess disability which is valid for assessment of disability (Guermazi et al., 2004) . The WOMAC consists of 9 items to assess physical function: rising from sitting, standing, bending to floor, walking on flat, shopping, putting on / taking off socks, rising from bed ,sitting(Guermazi et al., 2004)

\section{Procedures}

- The goal of the study and the method was described to all patients and inform consent were be taken prior to participation in the study. The potential benefits and inconveniences of all aspects of the study were clearly stated to participants. A meeting with patients was held to explain the purpose and protocols of the study; if patients chose to participate, they were complete the procedure.

- All patients participated in this study suffered from knee osteoarthritis diagnosed by the orthopedic surgeon through physical examination, X- ray, and MRI.

- All subjective data were collected and recorded in a specially designed sheet including Age, sex, weight, height and BMI.

- The three scales were given to the patients to read and answer each question by themself.

\section{Statistical analysis}

Before start of the study and based on the rule of thumb, sample size was calculated such as 5 to 10 participants per item. The data was collected by using statistical SPSS Package program version 23 for Windows (SPSS, Inc., Chicago, IL). The current test involved one independent variable was the (tested group); between subjects factor which had two levels (grade II of knee osteoarthritis group and grade III of knee osteoarthritis group). In addition, this test involved ten tested dependent variables (FSS, WOMAC, physical function, physical health, emotional problem, fatigue, emotional well-being, social function, pain and general health).

Prior to final analysis, data were screened for normality assumption, linearity, and presence of extreme scores. This exploration was done as a pre-requisite for parametric calculations of the analysis of difference. There was no a linear relationship between the dependent variables, as assessed by 
scatterplot. There were univariate outliers in the data, as assessed by inspection of a boxplot. Normality test of data using Kolmogorov-Smirnov test (used when sample size more than 50) was used, that reflect the data was not normally distributed for all dependent variables $(\mathrm{p}<0.05)$ at grade III group as well as using Shapiro-Wilk test (used when sample size less than $50)$ reflected that the data was not normally distributed $(\mathrm{p}<0.05)$ at grade II group. All these findings allowed the researchers to conduct non-parametric analysis. So, "Mann-Whitney U test " was used to compare FSS, WOMAC, physical function, physical health, emotional problem, fatigue, emotional well-being, social function, pain and general health between both groups. The alpha level was set at 0.05 . Statistical analysis was conducted using SPSS for windows, version 23 (SPSS, Inc., Chicago, IL).

\section{RESULTS}

\section{1 - General chronological features of the patients:}

The mean values of age, body mass and height were 56.6 \pm 4.07 years, $84.51 \pm 13.49 \mathrm{~kg}$ and $160.97 \pm 96.32 \mathrm{~cm}$ respectively as presented at table (1).

Table (1): Physical characteristics of participants in the study.

\begin{tabular}{|l|c|c|c|c|}
\hline Demographic variables & Minimum & Maximum & Mean & Std. Deviation \\
\hline Age & 50 & 65 & 56.6 & 4.07 \\
\hline Height & 145 & 175 & 160.97 & 6.32 \\
\hline Weight & 52 & 115 & 84.51 & 13.49 \\
\hline
\end{tabular}

\section{General Characteristics:}

The current study was conducted on 200 patients (166 females and 34 males) suffering from knee osteoarthritis of both sides. They were assigned according to the grade of OA into two groups. Grade II group consisted of 39 patients with mean age, body mass, and height values of $54.12 \pm 3.57$ years, $84.9 \pm 11.9 \mathrm{~kg}$, and $162.66 \pm 5.19 \mathrm{~cm}$ respectively. Grade III group consisted of 161 patients with mean age, body mass, and height values of $55.19 \pm 3.96$ years, $84.4 \pm 13.97 \mathrm{~kg}$, and $160.51 \pm 6.55 \mathrm{~cm}$ respectively. As indicated by the independent $t$ test, there were no significant differences ( $p>0.05)$ in the mean values of age, body mass, and height between both tested groups (Table2). The sex distribution of grade II group revealed that there were 36 females with reported percentage of $92.3 \%$ and 3 males with reported percentage of $7.7 \%$. The sex distribution of grade III group revealed that there were 131 females with reported percentage of $80.7 \%$ and 30 males with reported percentage of $19.3 \%$ as shown in table (2). Chi square revealed there was no significant differences between both groups in sex distribution $(\mathrm{p}>0.05)$ 
Table (2): Physical characteristics of patients in both groups.

\begin{tabular}{|c|c|c|c|c|c|}
\hline \multirow[t]{2}{*}{ Items } & \multirow{2}{*}{$\begin{array}{c}\text { Grade II } \\
\text { Mean } \pm \text { SD }\end{array}$} & \multirow{2}{*}{$\begin{array}{c}\text { Grade III } \\
\text { Mean } \pm \text { SD }\end{array}$} & \multicolumn{2}{|c|}{ Comparison } & \multirow[b]{2}{*}{$\mathbf{S}$} \\
\hline & & & t-value & P-value & \\
\hline Age (years) & $54.12 \pm 3.57$ & $55.19 \pm 3.96$ & -0.75 & 0.460 & NS \\
\hline Body mass (Kg) & $84.9 \pm 11.9$ & $84.4 \pm 13.97$ & 0.149 & 0.882 & NS \\
\hline Height (cm) & $162.66 \pm 5.19$ & $160.51 \pm 6.55$ & 1.387 & 0.169 & NS \\
\hline \multicolumn{6}{|c|}{ Sex distribution $N(\%)$} \\
\hline & Grade II & Grade III & $\mathbf{X}^{2}$ & P-value & NS \\
\hline Female & $36(92.3 \%)$ & $130(80.7 \%)$ & \multirow[t]{2}{*}{2.975} & \multirow{2}{*}{0.085} & \multirow[t]{2}{*}{ NS } \\
\hline Males & $3(7.7 \%)$ & $31(19.3 \%)$ & & & \\
\hline
\end{tabular}

*SD: standard deviation, P: probability, S: significance, NS: non-significant.

\section{Fatigue severity scale:}

As illustrated in figure (1), between groups comparison the median interquartile range (IQR) of the FSS in the "grade II group" was 36 (15). While, in the "grade III group" was 43 (10). Mann-Whitney U tests revealed that there was significant difference in the FSS between both groups with $\left(\mathrm{U}\right.$-value $=2019, \mathrm{Z}$-value $=\mathbf{- 3 . 4 5 8}, \mathrm{p}=0.001^{*}$ ) and this significant increase in favor to grade III group in compared to grade II group.

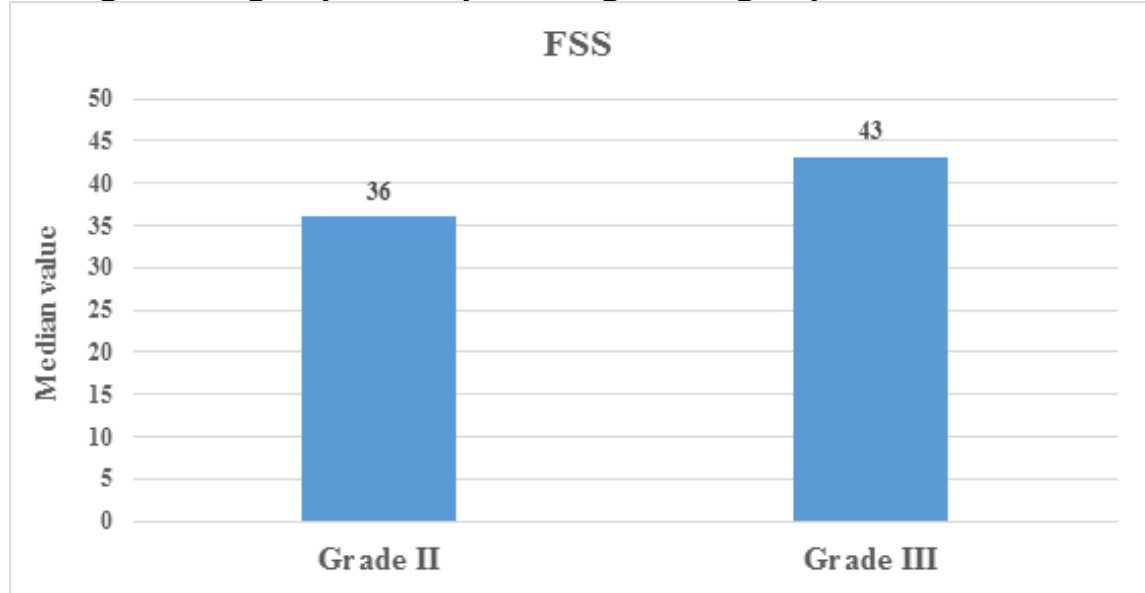

Figure (1): Median value of FSS between both groups.

\section{Correlation study}

1. Correlation between the mean value of FSS and WOMAC:

Spearman's correlation coefficient ( $\boldsymbol{\rho}$ ) between mean value of FSS $(41.86 \pm 9.54)$ and WOMAC $(18.83 \pm 5.17)$ was 0.263 . The results indicated that there was a positive weak correlation $(\mathrm{p}=0.0001 *)$. This means that increase in the FSS is consistent with increase in WOMAC (fig 2). 


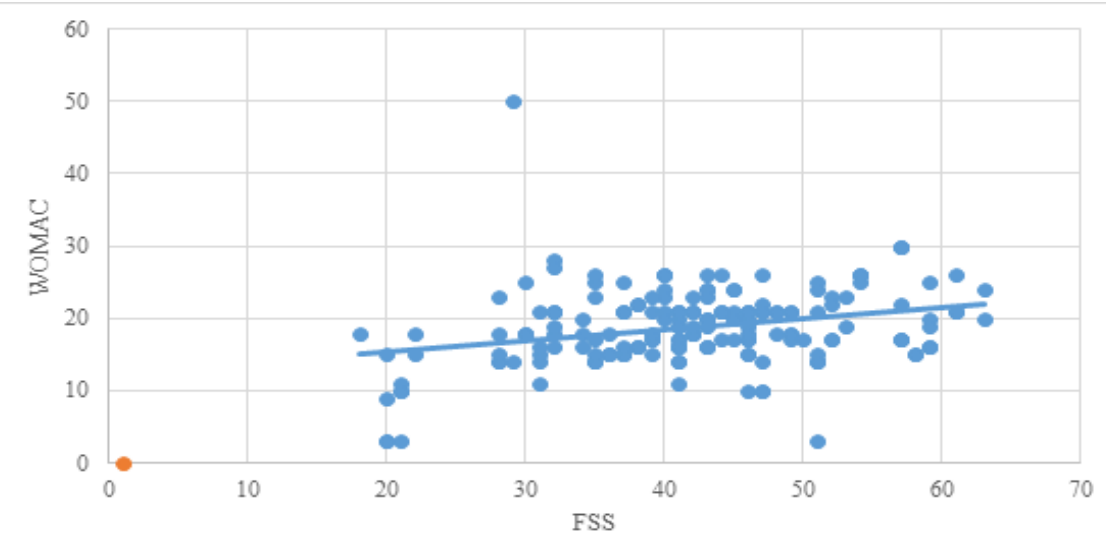

Figure (2). Scatter plot for the bivariate correlation between FSS and WOMAC.

\section{Physical health Domain of SF-36 questioner:}

2. Correlation between the mean value of FSS and physical functioning:

Spearman's correlation coefficient ( $\boldsymbol{\rho})$ between the mean value of FSS (41.86 \pm 9.54$)$ and physical functioning (41.59 \pm 14.55$)$ was -0.393 . The results indicated that there was a negative weak correlation $\left(\mathrm{p}=0.0001^{*}\right)$. This means that increase in the FSS is consistent with decrease in physical function (fig 3).

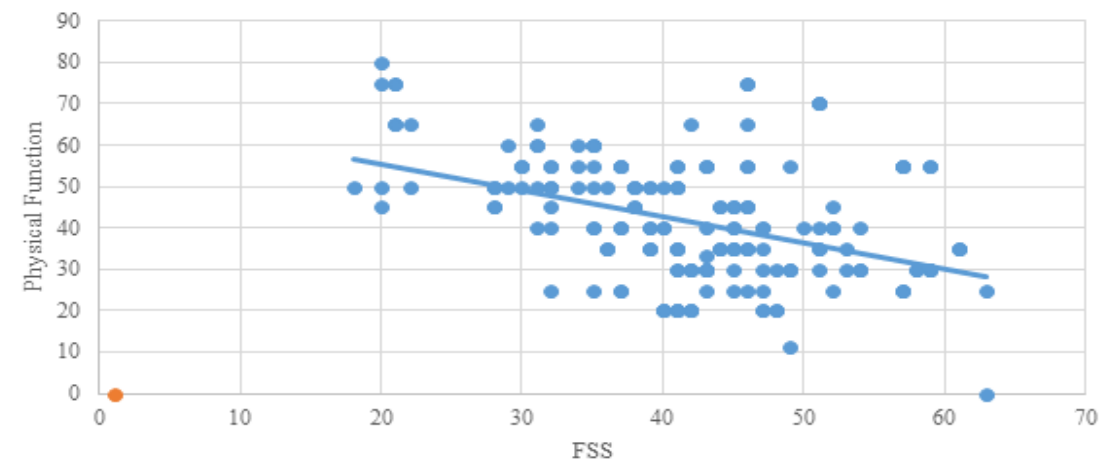

Figure (3). Scatter plot for the bivariate correlation between FSS and physical function.

3. Correlation between the mean value of FSS and role physical :

Spearman's correlation coefficient ( $\boldsymbol{\rho})$ between mean value of FSS $(41.86 \pm 9.54)$ and role physical $(18.5 \pm 22.16)$ was -0.052 . The results indicated that there was no correlation $(\mathrm{p}=0.468)$. This means that change in the FSS is inconsistent with change in role physical (fig 4). 


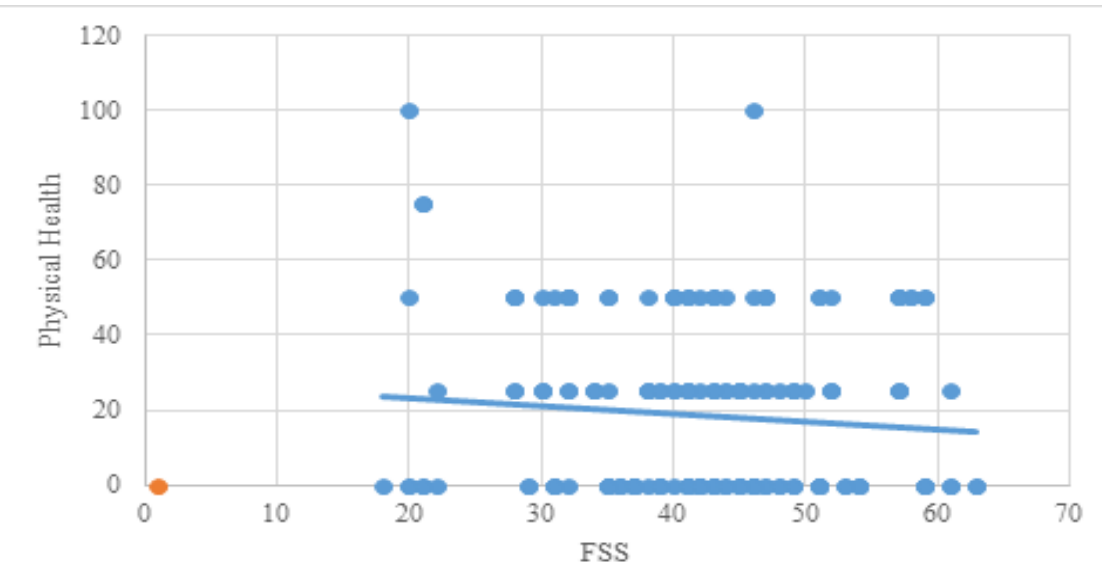

Figure (4). Scatter plot for the bivariate correlation between FSS and role physical .

4. Correlation between the mean value of FSS and pain domain:

Spearman's correlation coefficient $(\boldsymbol{\rho})$ between mean value of FSS $(41.86 \pm 9.54)$ and pain domain $(39.07 \pm 14.08)$ was -0.437 . The results indicated that there was a negative weak correlation $\left(\mathrm{p}=0.0001^{*}\right)$. This means that increase in the FSS is consistent with decrease in pain domain (fig5).

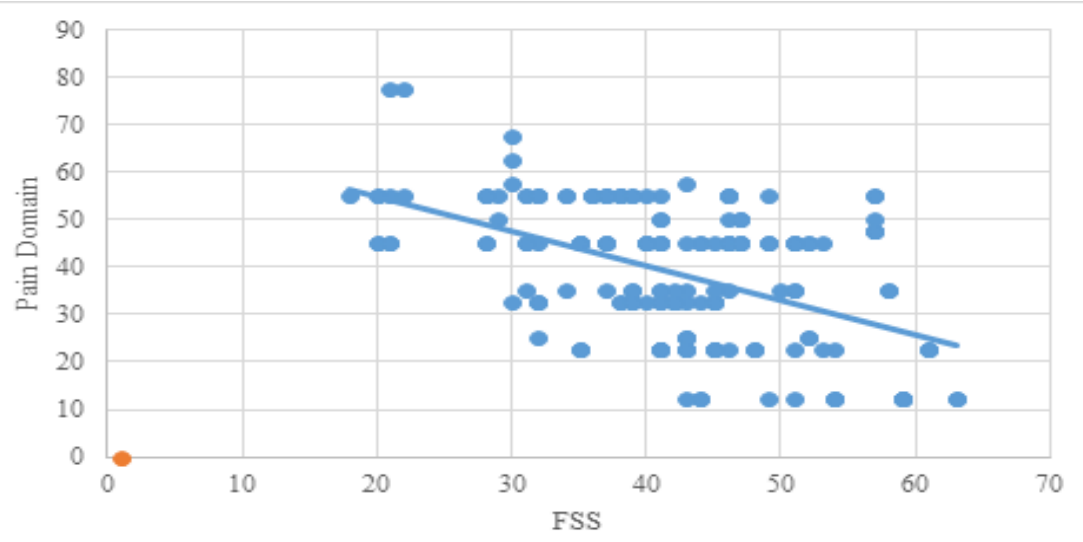

Figure (5). Scatter plot for the bivariate correlation between FSS and pain domain.

5. Correlation between the mean value of FSS and general health:

Spearman's correlation coefficient ( $\boldsymbol{\rho}$ ) between mean value of FSS $(41.86 \pm 9.54)$ and general health domain $(40.49 \pm 9.89)$ was -0.143 . The 
results indicated that there was a negative weak correlation $\left(\mathrm{p}=0.044^{*}\right)$. This means that increase in the FSS is consistent with decrease in general health (fig6).

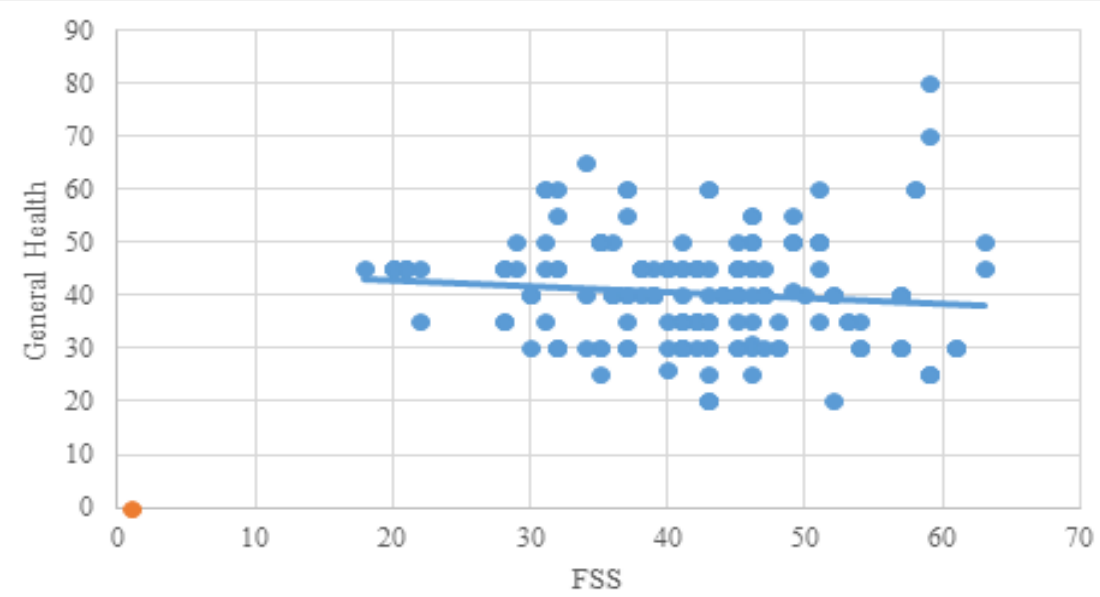

Figure (6). Scatter plot for the bivariate correlation between FSS and general health.

\section{Mental health domain of SF-36 questioner}

6. Correlation between the mean value of FSS and vitality domain:

Spearman's correlation coefficient $(\boldsymbol{\rho})$ between mean value of FSS $(41.86 \pm 9.54)$ and vitality domain $(35.55 \pm 14.13)$ was -0.213 . The results indicated that there was a negative weak correlation $(\mathrm{p}=0.002 *)$. This means that increase in the FSS is consistent with decrease in vitality domain (fig7).

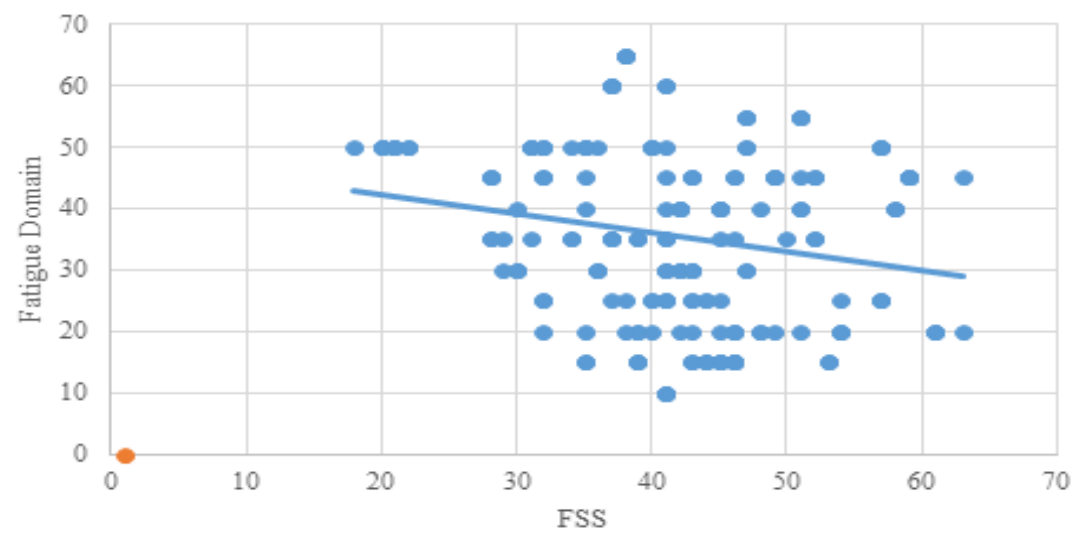

Figure (7). Scatter plot for the bivariate correlation between FSS and vitality domain. 
7. Correlation between the mean value of FSS and social function:

Spearman's correlation coefficient ( $\boldsymbol{\rho})$ between mean value of FSS $(41.86 \pm 9.54)$ and social function $(46.11 \pm 12.93)$ was -0.087 . The results indicated that there was no correlation $(\mathrm{p}=0.223)$. This means that change in the FSS is inconsistent with change in social function (fig8).

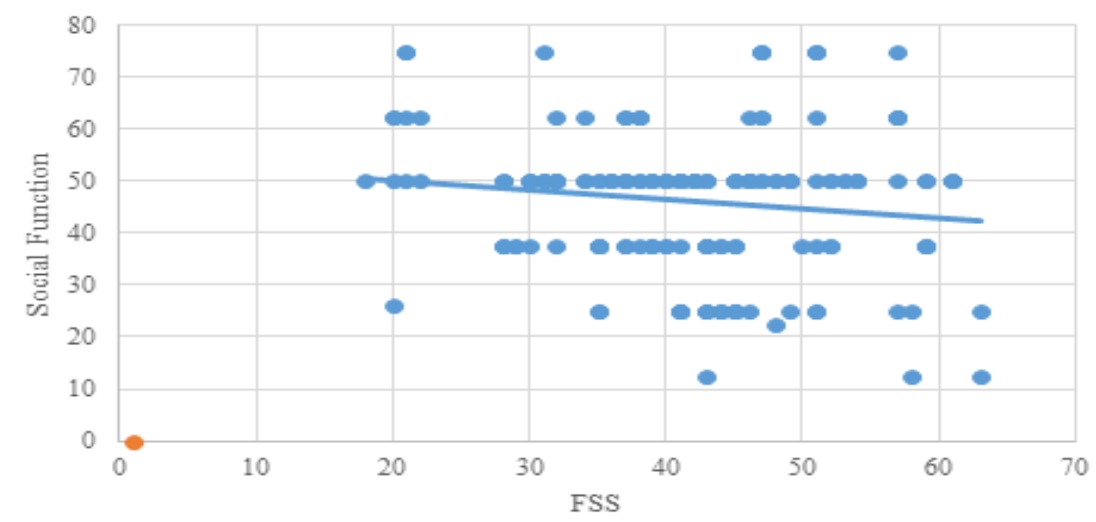

Figure (8). Scatter plot for the bivariate correlation between FSS and social function.

8. Correlation between the mean value of FSS and role motional:

Spearman's correlation coefficient ( $\rho$ ) between mean value of FSS $(41.86 \pm 9.54)$ and role emotional $(16.16 \pm 22.9)$ was 0.015 . The results indicated that there was no correlation $(\mathrm{p}=0.831)$. This means that change in the FSS is inconsistent with change in role emotional (fig9).

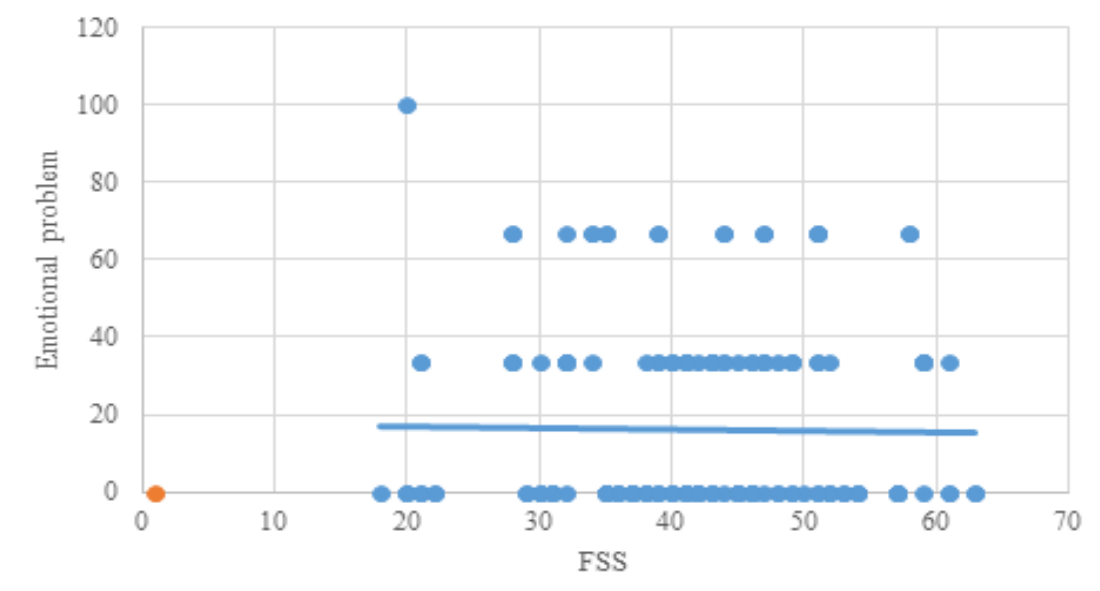

Figure (9). Scatter plot for the bivariate correlation between FSS and role emotional. 


\section{Correlation between the mean value of FSS and Mental health:}

Spearman's correlation coefficient ( $\boldsymbol{\rho})$ between mean value of FSS (41.86 \pm 9.54$)$ and mental health $(47.84 \pm 16.58)$ was 0.123 . The results indicated that there was no correlation $(\mathrm{p}=0.083)$. This means that change in the FSS is inconsistent with change in mental health (fig10).

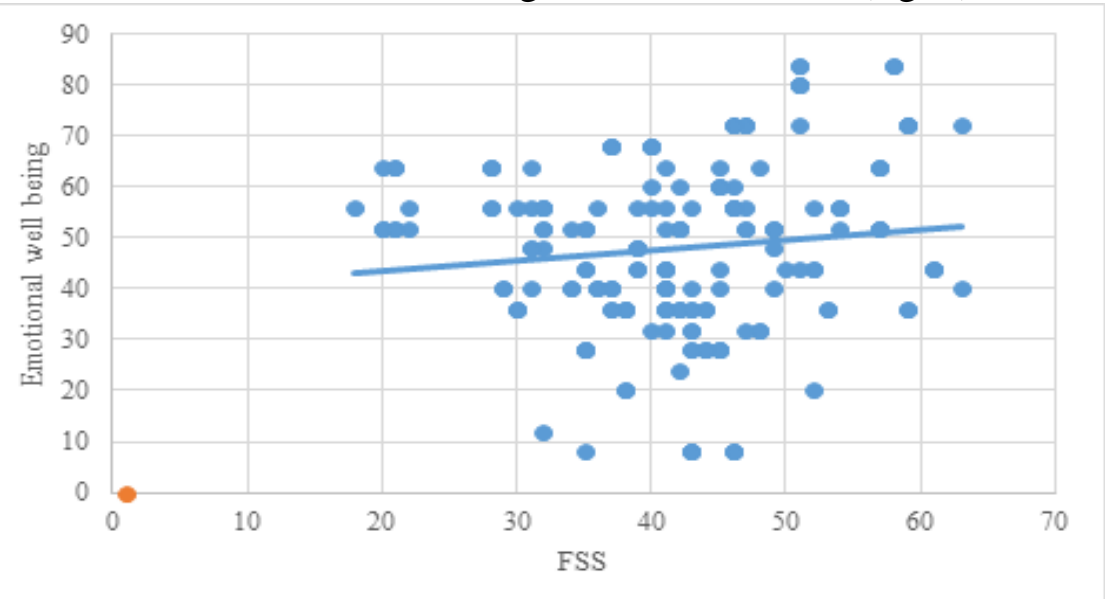

Figure (10). Scatter plot for the bivariate correlation between FSS and mental health.

\section{DISCUSSION}

This study was conducted to evaluate the fatigue and its association with both disability and quality of life in patients with knee osteoarthritis. The current study was conducted on 200 patients suffering from knee osteoarthritis of both sides. They were assigned into two studies groups' according to grade of OA. Mann-Whitney U tests revealed that there was a significant difference in the Fatigue severity scale between grade II and III of knee OA and this significant increase into word grade IIII group in compared to grade II group. There was a positive weak correlation between the mean value of FSS and WOMAC and a negative weak correlation between FSS and different domains of SF-36 included physical functioning, pain, vitality and general health domain. While there was no correlation between the mean value of FSS and role physical, role emotional, mental health, and social domain of SF-36.

Fatigue occurs as a result of a complicated interaction of medical, physical, and psychiatric factors (Doris et al., 2010). Tulay \& Fatma, (2019) was found that individuals with Knee OA experience more fatigue, insomnia and depression than in healthy individuals. Also founded that the participants with Knee OA experienced high levels of fatigue. In Other study, it has been determined that individuals with OA experience fatigue, sleeping problems and depression more than healthy individuals (Vitiello et al., 2014). The individuals 
in the two studies experienced fatigue; however, the level of fatigue varied from one study to another, which might be due to differences in the measurement tools used or due to their cultural characteristics. Snijders et al., 2011 confirms that fatigue, and even severe fatigue, is highly prevalent among patients with knee and/or hip OA. Power et al., 2008 found that individual with OA have significant amounts of fatigue and indicated that it had a substantial impact on their lives.

This study found that fatigue affect patient with knee OA and there was significant increase toward grade III group in compared to grade II group.

The study found also that there was a positive correlation between fatigue and functional disability. this results are similar to Garip et al., (2016) results which found that intensity and duration of fatigue, and interference with quality of life subgroups of FSI were found to be positively correlated with WOMAC A, WOMAC B, WOMAC C, WOMAC total, VAS-pain, BDS, and pain, energy, and emotional reactions subgroups of NHP $(\mathrm{p}<0.05)$. Physical mobility and sleep subgroups of NHP were only correlated with intensity of fatigue and duration of fatigue $(\mathrm{p}<0.05)$. There was no correlation between FSI subgroups and social isolation subgroup of NHP ( $p>0.05)$ which reported that the knee osteoarthritic patients were fatigued mostly in the evenings (Garip et al., 2016).

Similarly, Allen et al., (2013) founded an association between pain subgroup of WOMAC and VAS-fatigue in the study where 291 patients with knee and hip OA were assessed. In this study the fatigue evaluated by using VAS-fatigue, which is inadequate in determining different facets of fatigue. On the other hand, Stebbings et al., (2010) assessed fatigue of OA patients in New Zealand by using Multidimensional Assessment of FatigueGlobal Fatigue Index and reported a statistically significant correlation between fatigue and physical disability. Also Wolfe et al., (1996) founded that pain was the strongest predictor of fatigue in OA, explaining $25 \%$ of the variance in fatigue scores in multivariate regression analyses.

This study found a negative weak correlation between fatigue and pain domains of SF-36 this differently from Tulay \& Fatma, (2019) determined that fatigue affected pain and there was a relationship between them. Similarly Murphy et al. 2008 found same results. In other study, it was also found that there was a relationship between pain and fatigue in patients with Knee OA (Garip et al., 2016).

Allen et al. 2013 used the VAS-fatigue and found a relationship between fatigue and pain in 348 patients. These results suggest that in the management of fatigue in individuals with Knee OA, pain is a factor that should be brought under control.

In the study of Yeşim et al., (2016) fatigue subgroups including intensity of fatigue and duration of fatigue were found to be associated with QoL in terms of pain, physical mobility, sleep, energy and psychological functions. Interference with QoL was correlated with only pain, energy and 
psychological functions. Social function domain of QoL was not linked with fatigue. This might be due to study sample involving mostly women which is similar to our study and there was no correlation between role physical, role emotional, mental health , and social domain of SF-36

In the study of Stebbings et al., 2010 that investigated the relationship between fatigue, sleep, pain and depression in patients with rheumatoid arthritis and $\mathrm{OA}$, the patients with OA were found to have more fatigue, sleep disturbance, pain and depression than the patients with rheumatoid arthritis. Patients with OA have also been shown to exhibit problems related to falling asleep, sleep care and early morning awakening (Parmelee et al., 2015). Relevant studies have also shown that there is an important relationship between fatigue and sleep disturbances (Stebbings et al., 2010)

Similar results obtained from Doris et al., 2010 suggest that application of therapeutic approaches to sleep disturbances in OA management would be an appropriate intervention to control fatigue in these patients.

Salaffi et al. 1991, as well as Marks R 2014 found that patients' pain experience and disability scores were strongly influenced by the presence of depressive symptoms. This linkage of pain, depression, and osteoarthritis disability observed by Salaffi et al. 1991is problematic as it is strongly associated with activity avoidance, a wide array of emergent cognitive issues, such as learned helplessness, job dissatisfaction, and an adverse disease outcome (Okma-Keulen and Hopman, 2001).

That is, Marks R 2014 stated that the bulk of the research on osteoarthritis research reveals a consistent picture of potentially debilitating overlapping symptoms, including a low sense of morale, social isolation, helplessness, further depression, anxiety, sleep disturbances, and disability that can heighten and prolong the osteoarthritic pain experience with few comprehensive treatment options. And that fostering a positive, rather than ignoring any negative affect, may help to attenuate the prevailing degree of osteoarthritic pain and disability (Keefe et al. 1990)

Zautra and Smith, 2001 stated that this negative series of feedback responses where depression, anxiety and coping ability are significantly correlated with the osteoarthritic patient's pain and disability levels potentially produces a vicious cycle of excess pain and disability, plus varying degrees of negative affect, regardless of the prevailing degree of osteoarthritic damage.

In this respect, McBeth et al., 2014 founded evidence from the chronic pain literature strongly suggests central nervous system influences of emotions and cognitions including individual behavioral characteristics along with psychosocial factors, and increased sensitivity to pain signals by the brain should not be ignored as potent factors that can mediate or moderate osteoarthritis outcomes in older adults.

Predictors of new-onset widespread pain in older adults: results from a population-based prospective cohort study in the UK. That is, it can be assumed that the perceptions, interpretations, and reactions of the affected individual to 
their impairment will interact with peripheral pain processes to produce or heighten the painful experience, as well as the extent of prevailing disability, thus heightening the changes of incurring reactive depression, even if there is little or no distinctive prevailing painful source that can be demonstrated objectively (Sofat et al. 2013).

\section{REFERENCES}

Abdulaziz, A.S. ; G. Joel; D. Jolaine and H.Ron (1998): Transalation of the RAND 36 Item Health Survey into Arabic. Medical Care., 36: 251-443.

Al-Sobayel, H. ; H. Al-Hugail ; R. Alsaif ; N. Albawardi ; A.H. Alnahdi ; A. Daif and et al. (2016): Validation of an Arabic version of Fatigue Severity scale. Saudi Med J.,37(1):73-8.

Alkan, B. ; F. Fidan ; A. Tosun and Ö. Ardıçoğlu(2014): Quality of life and selfreported disability in patients with knee osteoarthritis. Mod Rheumatol., 24 (1): 166-71.

Allen, K. ; H. Bosworth ; C. Coffman ; A. Jeffreys ; E. Oddone and W. Yancy (2013): Predictors of fatigue in patients with hip and knee osteoarthritis. Osteoarthritis Cartilage., 21: S250.

Araujo, I. ; M. Castro ; C. Daltro and M. Matos (2016): Quality of Life and Functional Independence in Patients with Osteoarthritis of the Knee. Knee Surg Relat Res.,28(3):219-24.

Bergman, M. ; S. Shahouri ; T. Shaver ; J. Anderson ; D. Weidensaul ; R. Busch ; S. Wang and F. Wolfe (2009). Is Fatigue an Inflammatory Variable in Rheumatoid Arthritis (RA)? Analyses of Fatigue in RA, Osteoarthritis, and Fibromyalgia. J. Rheumatol., 36: 2788-2794.

Dawson, J. ; L. Linsell ; K. Zondervan ; P. Rose ; T. Randall ; A. Carr and et al. (2004): Epidemiology of hip and knee pain and its impact on overall health status in older adults. Rheumatology.,43(4):497-504.

Dekker, J. ; P. Tola ; G Aufdemkampe and M. Winckers (1993): Negative affect, pain and disability in osteoarthritis patients: the mediating role of muscle weakness. Behav Res Ther., 31(2):203-6.

Fertelli, T. and F. Tuncay (2019): Fatigue in individuals with knee osteoarthritis: Its relationship with sleep quality, pain and depression. Pak J Med Sci., 35(4).

Guermazi, M. ; S. Poiraudeau ; M. Yahia ; M. Mezganni ; J. Fermanian ; M.H. Elleuch and et al. (2004): Translation, adaptation and validation of the Western Ontario and McMaster Universities osteoarthritis index (WOMAC) for an Arab population: The Sfax modified WOMAC. Osteoarthritis Cartilage.;12(6):459-68.

Garip, Y. ; T. Güler and Ö. Tuncer (2016): Fatigue Among Elderly Patients with Knee Osteoarthritis and Its Association with Functional Status, Depression and Quality of Life. Ank Med J., 16(1):0-0.

Kao, M.J. ; M.P. Wu ; M.W. Tsai ; W.W. Chang and S.F. Wu (2012):The effectiveness of a self-management program on quality of life for knee osteoarthritis (OA) patients. Arch Gerontol Geriatr., 54(2):317-24. 
Kawano, M. ; I. Araújo ; M. Castro and M. Matos (2015): Assessment of quality of life in patients with knee osteoarthritis. Acta Ortop Bras., 23(6):307-10.

Keefe, F. ; D. Caldwell ; D. Williams ; K. Gil ; D. Mitchell ; C. Robertson and et al. (1990): Pain coping skills training in the management of osteoarthritic knee pain: A comparative study. Behav Ther., 21(1):49-62.

Mortada, M. ; A. Abdul-Sattar and L. Gossec (2015): Fatigue in Egyptian patients with rheumatic diseases: A qualitative study. Health Qual Life Outcomes.; 13(1):1-8.

McBeth, J. ; R. Lacey and R. Wilkie (2014): Predictors of new-onset widespread pain in older adults: results from a population-based prospective cohort study in the UK. Arthritis Rheumatol Hoboken NJ.;66(3):757-67.

Murphy, S. ; D. Smith ; D. Clauw and N. Alexander (2008): The impact of momentary pain and fatigue on physical activity in women with osteoarthritis. Arthritis Rheum.,59(6):849-56.

Okma-Keulen, P. and M.Hopman-Rock (2001). The onset of generalized osteoarthritis in older women: A qualitative approach. Arthritis Care Res., 45: $183-190$.

Perruccio, A. ; R. Gandhi ; J. Lau; K. Syed; N. Mahomed and Y. Rampersaud (2016): Cross-sectional contrast between individuals with foot/ankle vs knee osteoarthritis for obesity and low education on health-related quality of life. Foot Ankle Int.;37(1):24-32.

Power, J. ; E. Badley ; M. French ; A. Wall and G. Hawker (2008): Fatigue in osteoarthritis: A qualitative study. BMC Musculoskelet Disord.;9:1-8.

Parmelee, P. ; C. Tighe and N. Dautovich (2015): Sleep disturbance in osteoarthritis: linkages with pain, disability, and depressive symptoms. Arthritis Care Res., 67(3):358-65.

Snijders, G. ; C. van den Ende ; J. Fransen ; P. van Riel ; M. Stukstette ; K. Defoort and et al. (2011): Fatigue in knee and hip osteoarthritis: the role of pain and physical function. Rheumatology.;50(10):1894-900.

Stebbings, S. ; P. Herbison ; T. Doyle ; G. Treharne and J. Highton (2010): A comparison of fatigue correlates in rheumatoid arthritis and osteoarthritis: disparity in associations with disability, anxiety and sleep disturbance. Rheumatology., 49(2):361-7.

Salaffi, F. ; F. Cavalieri ; M. Nolli and G. Ferraccioli (1991): Analysis of disability in knee osteoarthritis. Relationship with age and psychological variables but not with radiographic score. J Rheumatol.,18(10):1581-6.

Sofat, N. ; C. Smee ; M. Hermansson ; M. Howard ; E. Baker ; F. Howe and et al. (2013): Functional MRI demonstrates pain perception in hand osteoarthritis has features of central pain processing. J Biomed Graph Comput., 3(4).

Stebbings, S. ; P. Herbison ; T. Doyle; G. Treharne and J. Highton (2010): A comparison of fatigue correlates in rheumatoid arthritis and osteoarthritis: disparity in associations with disability, anxiety and sleep disturbance. Rheumatology., 49(2):361-7. 
Vitiello, M. ; S. McCurry ; S. Shortreed ; L. Baker ; B. Rybarczyk ; F. Keefe and et al. (2014): Short-term improvement in insomnia symptoms predicts long-term improvements in sleep, pain, and fatigue in older adults with comorbid osteoarthritis and insomnia. Pain.,155(8):1547-54.

WOMAC, (2004): Western Ontario and McMaster Universities osteoarthritis index (WOMAC) for an Arab population: The Sfax modified WOMAC. Osteoarthritis Cartilage.;12(6):459-68.

Wolfe, F. ; D. Hawley and K. Wilson (1996): The prevalence and meaning of fatigue in rheumatic disease. J Rheumatol., 23(8):1407-17.

Yu, D. ; D. Lee and N. Man(2010): Fatigue among older people: a review of the research literature. Int J Nurs Stud., 47(2):216-28.

Zautra, A. and B. Smith (2001): Depression and reactivity to stress in older women with rheumatoid arthritis and osteoarthritis. Psychosom Med., 63(4):687-96.

Zautra, A. ; R. Fasman ; B. Parish and M. Davis (2007): Daily fatigue in women with osteoarthritis, rheumatoid arthritis, and fibromyalgia. Pain., 128 (1): 128-35.

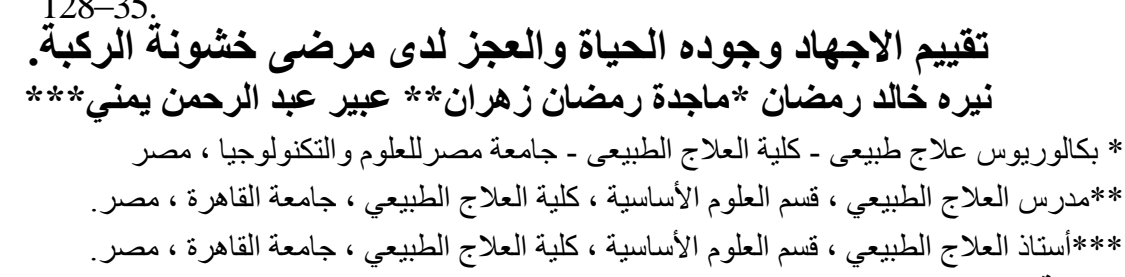

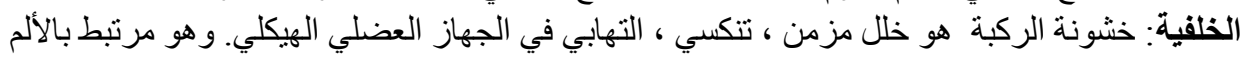

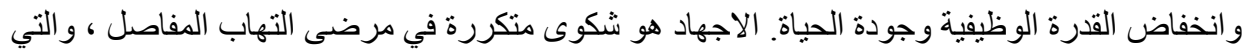

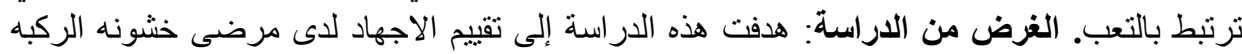

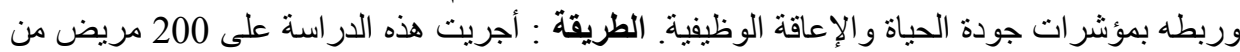

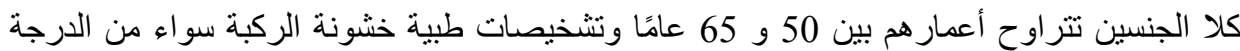

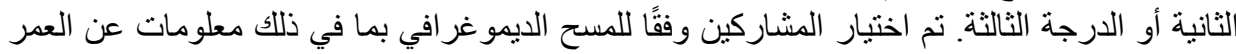

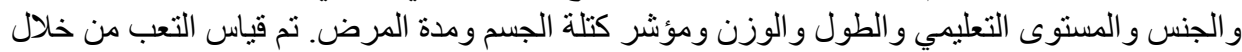

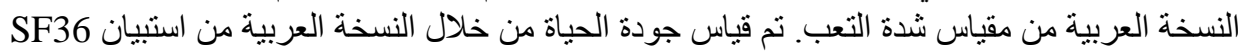

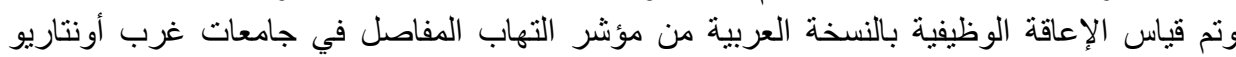

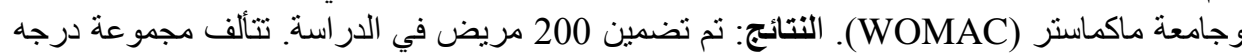

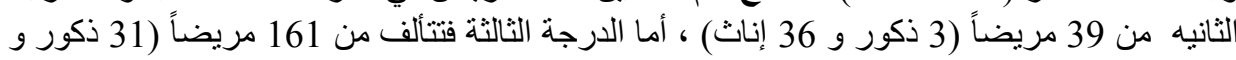

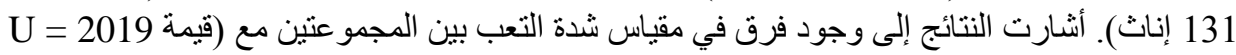

، قيمة

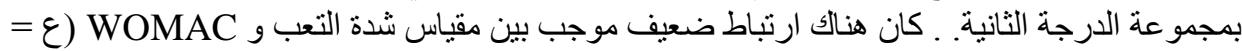

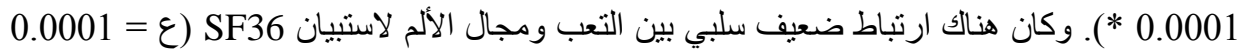

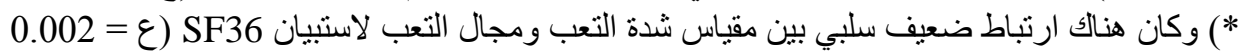

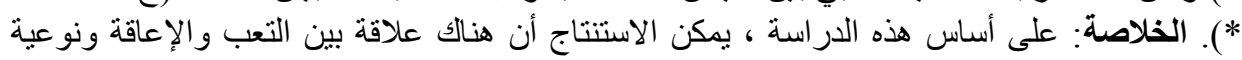

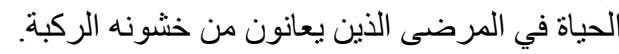

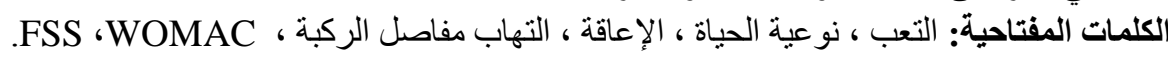

